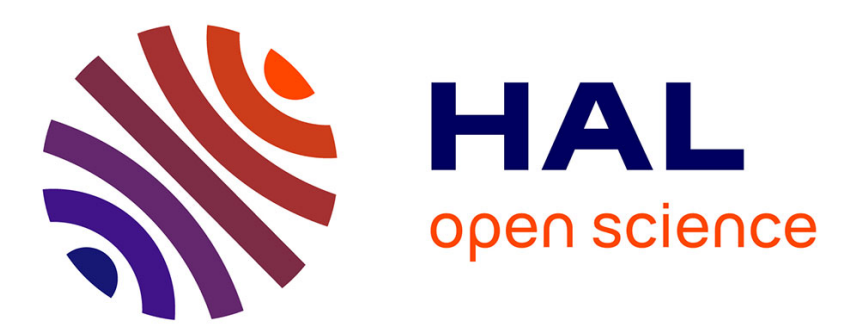

\title{
Sample collection from asteroid (162173) Ryugu by Hayabusa2: Implications for surface evolution
}

T. Morota, Seiji Sugita, Y. Cho, Masanori Kanamaru, Eri Tatsumi, N. Sakatani, R. Honda, H. Kikuchi, M. Yamada, Y. Yokota, et al.

\section{- To cite this version:}

T. Morota, Seiji Sugita, Y. Cho, Masanori Kanamaru, Eri Tatsumi, et al.. Sample collection from asteroid (162173) Ryugu by Hayabusa2: Implications for surface evolution. Science, 2020, 368 (6491), pp.654-659. 10.1126/science.aaz6306 . hal-03085258

\section{HAL Id: hal-03085258 https://hal.science/hal-03085258}

Submitted on 8 Jan 2021

HAL is a multi-disciplinary open access archive for the deposit and dissemination of scientific research documents, whether they are published or not. The documents may come from teaching and research institutions in France or abroad, or from public or private research centers.
L'archive ouverte pluridisciplinaire HAL, est destinée au dépôt et à la diffusion de documents scientifiques de niveau recherche, publiés ou non, émanant des établissements d'enseignement et de recherche français ou étrangers, des laboratoires publics ou privés. 


\section{Title: Sample collection from asteroid 162173 Ryugu by Hayabusa2: implications for surface evolution}

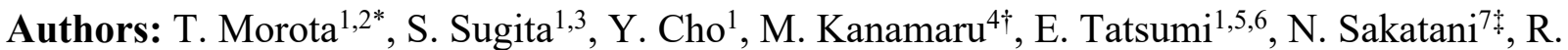

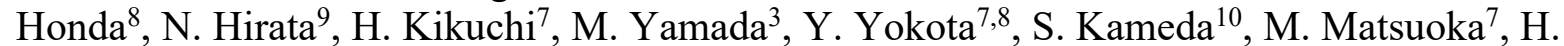
Sawada $^{7}$, C. Honda ${ }^{11}$, T. Kouyama ${ }^{12}$, K. Ogawa ${ }^{9,13}$, H. Suzuki ${ }^{14}$, K. Yoshioka ${ }^{1}$, M. Hayakawa ${ }^{7}$, N. Hirata ${ }^{11}$, M. Hirabayashi ${ }^{15}$, H. Miyamoto ${ }^{1,21}$, T. Michikami ${ }^{16}$, T. Hiroi ${ }^{17}$, R. Hemmi ${ }^{1}$, O. S. Barnouin $^{18}$, C. M. Ernst ${ }^{18}$, K. Kitazato ${ }^{11}$, T. Nakamura ${ }^{19}$, L. Riu ${ }^{7}$, H. Senshu ${ }^{3}$, H. Kobayashi ${ }^{2}$, S. Sasaki $^{4}$, G. Komatsu ${ }^{20}$, N. Tanabe ${ }^{1}$, Y. Fujii ${ }^{8}$, T. Irie $^{2}$, M. Suemitsu ${ }^{2}$, N. Takaki ${ }^{1}$, C. Sugimoto ${ }^{1}$,

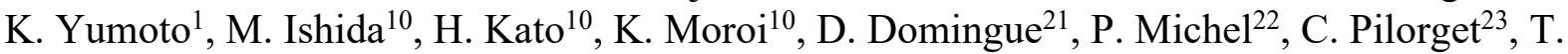

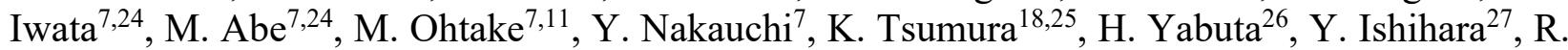
Noguchi $^{7}$, K. Matsumoto ${ }^{24,28}$, A. Miura ${ }^{7}$, N. Namiki ${ }^{24,28}$, S. Tachibana ${ }^{1}$, M. Arakawa ${ }^{9}$, H. Ikeda ${ }^{29}$, K. Wada ${ }^{3}$, T. Mizuno ${ }^{7,24}$, C. Hirose ${ }^{29}$, S. Hosoda ${ }^{7}$, O. Mori ${ }^{7}$, T. Shimada ${ }^{7}$, S. Soldini ${ }^{7,30}$, R.

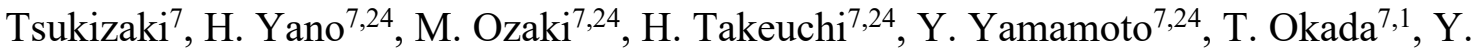
Shimaki $^{7}$, K. Shirai ${ }^{7}$, Y. Iijima ${ }^{7 \S}$, H. Noda ${ }^{24,28}$, S. Kikuchi ${ }^{7}$, T. Yamaguchi ${ }^{7 \#}$, N. Ogawa ${ }^{7}$, G.

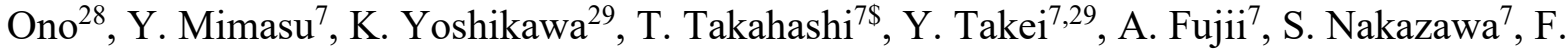

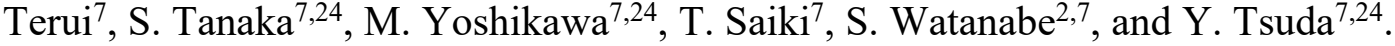

\section{Affiliations:}

1. The University of Tokyo, Tokyo 113-0033, Japan.

2. Nagoya University, Nagoya 464-8601, Japan.

3. Planetary Exploration Research Center, Chiba Institute of Technology, Narashino 275-0016, Japan.

4. Osaka University, Toyonaka 560-0043, Japan.

5. Departamento de Astrofísica, Universidad de La Laguna, 38206 La Laguna, Tenerife, Spain.

6. Instituto de Astrofísica de Canarias, 38205 La Laguna, Tenerife, Spain

7. Institute of Space and Astronautical Science, Japan Aerospace Exploration Agency (JAXA), Sagamihara 252-5210, Japan.

8. Kochi University, Kochi 780-8520, Japan.

9. Kobe University, Kobe 657-8501, Japan.

10. Rikkyo University, Tokyo 171-8501, Japan.

11. University of Aizu, Aizu-Wakamatsu 965-8580, Japan.

12. National Institute of Advanced Industrial Science and Technology, Tokyo 135-0064 Japan.

13. JAXA Space Exploration Center, Japan Aerospace Exploration Agency, Sagamihara 2525210, Japan.

14. Meiji University, Kawasaki 214-8571, Japan.

15. Auburn University, Auburn, AL 36849, USA.

16. Kindai University, Higashi-Hiroshima 739-2116, Japan.

17. Brown University, Providence, RI 02912, USA.

18. Johns Hopkins University Applied Physics Laboratory, Laurel, MD 20723, USA.

19. Tohoku University, Sendai 980-8578, Japan.

20. International Research School of Planetary Sciences, Università d'Annunzio, 65127 Pescara, Italy.

21. Planetary Science Institute, Tucson, AZ 85719-2395, USA. 
22. Université Côte d'Azur, Observatoire de la Côte d'Azur, Centre National de le Recherche Scientifique, Laboratoire Lagrange, 06304 Nice, France.

23. Institut d'Astrophysique Spatiale, Université Paris-Sud, Orsay, France.

24. SOKENDAI (The Graduate University for Advanced Studies), Hayama 240-0193, Japan.

25. Tokyo City University, Tokyo 158-8557, Japan.

26. Hiroshima University, Higashi-Hiroshima 739-8526, Japan.

27. National Institute for Environmental Studies, Tsukuba 305-8506, Japan.

28. National Astronomical Observatory of Japan, Mitaka 181-8588, Japan.

29. Research and Development Directorate, JAXA, Sagamihara 252-5210, Japan.

30. The University of Liverpool, Liverpool L69 3BX, UK.

${ }^{*}$ Corresponding author: E-mail: morota@eps.s.u-tokyo.ac.jp

${ }^{\dagger}$ Affiliation from April 2020: Institute of Space and Astronautical Science, Japan Aerospace Exploration Agency (JAXA), Sagamihara 252-5210, Japan. ¥Affiliation from April 2020: Rikkyo University, Tokyo 171-8501, Japan $\$$ Deceased. ${ }^{\#}$ Current affiliation: Mitsubishi Electric Corporation, Kamakura 247-8520, Japan. \$Current affiliation: NEC Corporation, 1-10 Nisshin-cho, Fuchu, Tokyo 183-0036, Japan.

Abstract: The near-Earth asteroid 162173 Ryugu is thought to be a primitive carbonaceous object and to contain hydrated minerals and organic molecules. We report sample collection from Ryugu's surface by the Hayabusa2 spacecraft on 21 February 2019. Touchdown images and global observations of surface colors are used to investigate the stratigraphy of the surface around the sample location and across Ryugu. Latitudinal color variations suggest reddening of exposed surface material by solar heating and/or space weathering. Immediately after touchdown, Hayabusa2's thrusters disturbed dark fine grains that originate from the redder materials. The stratigraphic relationship between identified craters and the redder material suggests that surface reddening occurred over a short period of time. We suggest that Ryugu has previously experienced an orbital excursion near the Sun.

\section{Main Text:}

Hayabusa2 is a sample-return mission to 162173 Ryugu, a carbonaceous near-Earth asteroid (NEA) (1). The spacecraft launched on 3 December 2014 and arrived at Ryugu on 27 June 2018. Initial global observations showed the asteroid has a spinning top-shape and rubblepile structure $(2,3)$. The surface has generally uniform spectra $(4,5)$. Small variations in surface color are quantified using the spectral slope from $b$-band $(0.48 \mu \mathrm{m})$ to $x$-band $(0.86 \mu \mathrm{m})$ (hereafter, $b$ - $x$ slope) $(2,5,6)$ observed with the telescopic optical navigation camera (ONC-T) (Fig. 1A) (5). Two compositional types of material are present on the surface with different colors: bluer material distributed at the equatorial ridge and in the polar regions, and redder material in the mid-latitude regions (5). However, the cause of these spectral variations is not understood.

On 21 February 2019, Hayabusa2 conducted its first sample collection from Ryugu, performing a touchdown on the surface. During the touchdown operation, Hayabusa2 took 
images of Ryugu's surface with resolutions as high as $\sim 1 \mathrm{~mm} \mathrm{pixel}^{-1}$. Those images allow us to observe the surface response to the physical disturbances generated by the touchdown, including the sampling projectile collision and firing of the thruster gas jets.

The touchdown site was selected based on established engineering safety criteria and scientific merits $(2,6,7)$. Regional variations in the mixing ratio between redder and bluer materials are measured using the spectral slope (Fig. 1A). Spectral variations between candidate touchdown sites are much smaller than those within each site (2), implying that the surface materials are locally well-mixed. A sampling operation at any of the candidate sites should therefore collect both redder and bluer components. Safe landing locations were limited by the high boulder abundances $(5,8)$. We initially intended the site designated L08-B, one of the lowest boulder density areas on Ryugu, as the primary landing site $(2,6,7)$ and deployed a target marker (TM) on October 23, 2018 to facilitate navigation. Based on the location where the TM settled and the detailed search for areas without boulders taller than $65 \mathrm{~cm}$, which could reach Hayabusa2's reaction control system (RCS) during a touchdown, we finally chose L08-E1 (Fig. 2A and S5).

Hayabusa2 performed multiple low-altitude $(\sim 40 \mathrm{~m})$ descent maneuvers near the L08 region, during which we conducted high-resolution $\left(>0.01 \mathrm{~m} \mathrm{pixel}^{-1}\right)$ spectral and morphological observations. The touchdown site is generally slightly bluer than the global average, but reddish areas are found within L08-E1 (Fig. 1E and 2C). These reddish areas are on average slightly darker than bluer areas (Fig. S6), a trend that is observed globally $(2,5)$. The reddish areas are limited to parts of individual boulders, with most boulder surfaces in this location being blue (Fig. 2C). These observations are compatible with the interpretation that Ryugu's boulders are originally bluer, with redder materials produced by surface metamorphic processes such as space weathering (9), thermal metamorphism by solar heating (10), and/or pulverization by small impacts (11). Redder materials may have been shed from boulder surfaces by impact disruption and/or thermal fatigue. The bluer surfaces of boulders remain un-reddened, implying that the timescale for surface reddening is longer than boulder resurfacing by impact disruption and/or thermal fatigue.

There are two morphological types of sub-meter-sized boulders around the touchdown site: dark ragged boulders and bright boulders with smooth surfaces (Fig. 2D and S7A). These types of boulders are observed in the ten-meter size range in remote images (5), and on smaller scales in images taken on the surface by the Mobile Asteroid Surface Scout (MASCOT) lander (12). Images taken during the descent operation show that there is also a submeter-scale heterogeneity in surface reflectance of the bright boulders: the edges of many boulders are brighter than the planar surfaces of the same boulders (yellow arrows in Fig. 2E and S7B). This may be due to a higher abrasion rate at the boulder edges. One boulder in Fig. 2E appears to have broken in two, with the possible broken surface $\sim 1.5$ times brighter than surfaces of the surrounding boulders. Because the interior/less-processed part of the boulder is exposed on the boulder edges and broken surface, these observations suggest that boulder surfaces are generally darkened by exposure to space.

The Hayabusa2 spacecraft has a sampling mechanism (13) similar to that of the original Hayabusa spacecraft (14). Ejecta are generated by the impact of a 1-cm-diameter tantalum projectile fired at a speed of $\sim 300 \mathrm{~m} \mathrm{~s}^{-1}$ during the first contact with the surface, which are caught with a sampler horn extended from the bottom of the spacecraft $(13,14,15)$.

The combination of the impact of the projectile fired from the sampler system and the operation of the RCS thrusters during the touchdown produced a large amount of debris from 
Ryugu's surface (Fig. 2G and Movie S1) (6). The video obtained by the nadir-viewing wideangle optical navigation camera (ONC-W1) indicates that large boulders (up to $1 \mathrm{~m}$ in the longest dimension) were moved horizontally by $>5 \mathrm{~m}$ during the touchdown (Fig. S9). However, most of the debris disturbed during the touchdown was small pebbles and fine grains whose diameters are less than the pixel size (which varied from 1 to a few $\mathrm{mm}$ ) of the W1 images (Fig. S8) (6). This high mobility of fragmental debris deposits (i.e., regolith) during the touchdown indicates that the cohesive forces between boulders/pebbles may be very weak, or that they depend on the particle sizes. This interpretation is consistent with the observed deficit of small craters $(5,16)$, mass movement along slopes (i.e., mass wasting) on crater walls $(5)$, and the crater formation by Hayabusa2's artificial impact experiment (17).

Immediately after the RCS thrust upon touchdown, the entire field-of-view of ONC-W1 was darkened uniformly. A dark ragged boulder, which we nickname "Turtle Rock" simultaneously became as bright as the surrounding brighter boulders (Fig. 2F, and 2H) (6). Turtle Rock was also moved by the RCS exhaust. These observations suggest that dark fine grains were originally present on the surfaces (or inside pores) of darker and redder boulders, before being lifted by the RCS thrusting. Such fine grains were not visible in the surface images taken by MASCOT (12). The sampling process produced a cloud of dark fine grains that expanded from the touchdown site and extended to a zone $\sim 10-\mathrm{m}$ in diameter, centered at the touchdown site (Fig. $2 \mathrm{G}$ and $2 \mathrm{H}$ ). We estimate the total mass of the fine-grained cloud to be $\sim 12$ $\mathrm{kg}$ (6). The pre-touchdown color of this region was slightly bluer than the surrounding region, but it became redder after the deposition of the lofted dark fine grains (Fig. 2G and S11) (6). Observations with the Near Infrared Spectrometer (NIRS3) of the region before and after the touchdown showed little change in the $\mathrm{OH}$ band depth, although the NIRS3 spectra after the touchdown is slightly bluer and brighter (Fig. S12). This is consistent with the global lack of correlation between the spatial distribution of the $\mathrm{OH}$ band depth and the red-blue color variations observed by $\mathrm{ONC}(4,5)$. We conclude that dark fine red grains originally concentrated on the boulder surfaces or in voids on the boulders, are not compositionally distinct from the subsurface, and the disruption by the touchdown event resulted in more even distribution of the grains across the affected area.

Globally, the $b-x$ slope varies with latitude (Fig. 1A). We found that the $b-x$ slope also correlates with the crater distribution. Fresh and stratigraphically younger craters $>20 \mathrm{~m}$ in diameter have interiors that are spectrally bluer than their surroundings (Fig. 1C). This implies that the redder materials were covering the bluer materials, with the latter exposed by the crater formation. This is consistent with the stratigraphic relationship between the redder and bluer materials inferred from the global $b-x$ slope distribution (5). On the other hand, stratigraphically older craters tend to have redder interiors, and the color of the crater interiors is similar to that of surrounding materials (Fig. 1C). We investigated the contrasts in spectral slopes between crater interior surfaces and surrounding areas, which we define as the area within a distance of one 40 crater radius outward from the crater rim (6). The histogram of the contrast in $b-x$ spectral slope shows a bimodal distribution (Fig. 3), indicating that craters on Ryugu can be divided into two groups: red craters whose interior has $b-x$ slope similar to that of their surroundings, and blue craters whose interior is bluer than their surroundings.

A potential explanation of the latitudinal variation in $b-x$ slope is that the exposure of Ryugu's materials to space has led to their reddening, and mass wasting from the equator and polar regions (topographic highs) to the mid-latitude regions (topographic lows) has exposed 
fresh bluer subsurface materials $(2,5)$. The polar regions exhibit bluer spectra than the equatorial ridge (Fig. S3), suggesting that the reddening process seems to depend on illumination by the Sun (Ryugu's obliquity is $171.6^{\circ}(2)$ ). The color variation of crater interiors can be explained by their stratigraphic relations; the craters with redder interiors were formed before surface reddening occurred, while the bluer craters were formed after the surface reddening and the underlying bluer materials were exposed by the impacts. The bimodal distribution of the contrast in $b-x$ spectral slope (Fig. 3) suggests that the surface reddening has not been active throughout the whole Ryugu's history and occurred mainly within a period after the formation of redder craters and before the formation of bluer craters. The surface reddening might not be currently active, or is active but slow compared with the resurfacing processes of boulder surfaces as discussed above (Fig. 2C).

We interpret the crater size-frequency distributions (SFDs) using collision frequency models derived for the asteroid main belt $(6,18,19)$. From the SFD of red craters larger than 100 $\mathrm{m}$ in diameter (Fig. 3D), we estimate the time between the formation of Ryugu to the surface reddening event to be $8.5 \mathrm{Ma}$. This is much younger than the breakup time of candidate parent asteroids, Eulalia and Polana (several hundred Ma to $\sim 1 \mathrm{Ga})(5,20)$, suggesting that Ryugu is the product of more than one generation of parent body disruption and/or global resurfacing processes such as the spinning top-shape formation had occurred until $8.5 \mathrm{Ma}$ ago. The observed number density of blue craters is $\sim 30$ times lower than that of red craters (Fig. 3D). We estimate a model age for the reddening event of about $0.3 \mathrm{Ma}$, from the observed SFD of blue craters larger than $30 \mathrm{~m}$ diameter based on the main-belt collision frequency model (Fig. S14A). Using an alternative NEA collision frequency model, the age of the reddening event is estimated to be about $8 \mathrm{Ma}$, because there is a much lower collision frequency for bodies in NEA orbits $(6,18$, 19). We interpret these ages as upper and lower limits of age estimates of the surface reddening. The NEA model age is younger than the typical dynamical lifetime of NEAs ( $10 \mathrm{Ma})(21)$ and the median orbital lifetime of Ryugu ( $40 \mathrm{Ma}$ ) (22). We therefore suggest that the reddening of Ryugu's surface occurred after its orbit shifted from the main belt to its current near-Earth orbit (5).

The deficit of craters smaller than $100 \mathrm{~m}$ in diameter on Ryugu's surface suggests that crater erasure processes have occurred (5). Existing small craters must therefore have formed geologically recently. However, smaller craters $(<10 \mathrm{~m}$ in diameter) do not always exhibit bluer interiors, which would be expected if they were all young. Some small craters exhibit a redder, not bluer, interior than the surroundings materials (Fig. 1E). Streaked patterns of redder materials such as ejecta deposits are visible across the whole surface (Fig. S4B). In addition, a streaked pattern of redder materials elongated from a boulder suggests that a mass of redder material collided with the boulder and dispersed (Fig. S4F). Redder materials may have been disrupted and redistributed by impacts, thermal fatigue, and mass wasting, which may have resulted in the formation of a mixed layer of redder and bluer materials after the surface reddening event (Fig. 4). This interpretation is supported by the distribution of redder materials on boulder surfaces 40 and the existence of dark reddish fine grains observed in the touchdown operation. The thickness of the mixed layer of redder and bluer materials is estimated to be a few meters, derived from the minimum crater size $(\sim 10 \mathrm{~m}$ in diameter) that penetrates to the underlying blue materials. The presence of ejecta rays with a length of a few tens of meters that consist primarily of redder materials (Fig. S4F) implies that the redder material layer originally had a minimum thickness of 45 a few tens of centimeters (6). Solar heating is more likely than space weathering to be the source of the reddening of Ryugu's surface, because space weathering typically affects only a thin layer 
of about $100 \mathrm{~nm}$, while the diurnal and annual thermal skin depths (depth at which temperature decays to $1 / \mathrm{e}$ the surface temperature) are $\lesssim 10 \mathrm{~cm}$ and $\sim 1.5 \mathrm{~m}$, respectively $(23,24)$.

We suggest that a surface reddening event within a short period of time could be explained if Ryugu underwent a temporary orbital excursion near the Sun, causing higher surface heating. Such solar heating is consistent with the apparent deficiency in C-type asteroids bearing aqueous alteration features at $0.7-\mu \mathrm{m}$ band in the NEA population (25). However, solar heating on Ryugu during an orbital excursion cannot account for the low abundance of hydrous minerals revealed by global observations $(4,5)$, because the bluish/brighter areas on Ryugu, which did not experience the intense solar heating, also have low abundance of hydrous minerals (4).

The large local variations in the spectral slope and albedo within the sampling site suggest that both bluer and redder components were likely collected during the touchdown. We predict that the returned sample will contain a mix of altered and unaltered materials, with the former recording a solar heating event.

\section{References and Notes:}

1. S. Watanabe et al., Hayabusa2 mission overview. Space Sci. Rev. 208, 3-16 (2017). doi: 10.1007/s11214-017-0377-1

2. S. Watanabe et al., Hayabusa2 arrives at the carbonaceous asteroid 162173 Ryugu-A spinning top-shaped rubble pile. Science 364, 268-272 (2019). doi:10.1126/science.aav8032

3. M. Hirabayashi et al., The western bulge of 162173 Ryugu formed as a result of a rotationally driven deformation Process. Astrophys. J. Lett. 874, L10 (2019). doi:10.3847/2041-8213/ab0e8b

4. K. Kitazato et al., Surface composition of asteroid 162173 Ryugu as observed by the Hayabusa2 NIRS3 instrument. Science 364, 272-275 (2019). doi:10.1126/science.aav7432

5. S. Sugita et al., The geomorphology, color, and thermal properties of Ryugu: Implications for parent-body processes. Science 364, eaaw0422 1-11 (2019). doi:10.1126/science.aaw0422

6. Materials and Methods are available as Supplementary Materials.

7. S. Kikuchi et al., Design and reconstruction of the Hayabusa2 precision landing on Ryugu. 2019 AAS/AIAA Astrodynamics Specialist Conference, AAS 19-762 (2019).

8. T. Michikami et al., Boulder size and shape distributions on asteroid Ryugu, Icarus 331, 179-191 (2019) doi:10.1016/j.icarus.2019.05.019

9. C. Lantz et al., Ion irradiation of carbonaceous chondrites: A new view of space weathering on primitive asteroids. Icarus 285, 43-57 (2017). .doi:10.1016/j.icarus.2016.12.019

10. T. Hiroi, M. E. Zolensky, C. M. Pieters, M. E. Lipschutz, Thermal metamorphism of the C, $\mathrm{G}, \mathrm{B}$, and $\mathrm{F}$ asteroids seen from the $0.7 \mu \mathrm{m}, 3 \mu \mathrm{m}$, and $\mathrm{UV}$ absorption strengths in comparison with carbonaceous chondrites. Meteorit. Planet. Sci. 31, 321-327 (1996). doi:10.1111/j.1945-5100.1996.tb02068.x

40 11. E. A. Cloutis, P. Hudon, T. Hiroi, M. J. Gaffey, Spectral reflectance properties of carbonaceous chondrites 4: Aqueously altered and thermally metamorphosed meteorites. Icarus 220, 586-617 (2012). doi: 10.1016/j.icarus.2012.05.018

12. R. Jaumann et al., Images from the surface of asteroid Ryugu show rocks similar to carbonaceous chondrite meteorites. Science 365, 817-820 (2019). doi:10.1126/science.aaw8627 
13. S. Tachibana et al., Hayabusa2: Scientific importance of samples returned from C-type nearEarth asteroid (162173) 1999 JU3. Geochemical J. 48, 571-587 (2014). doi:10.2343/geochemj.2.0350

14. H. Yano et al., Touchdown of the Hayabusa Spacecraft at the Muses Sea on Itokawa. Science 312, 1350-1353 (2006). doi:10.1126/science.1126164

15. H. Sawada et al., Hayabusa2 sampler: Collection of asteroidal surface material. Space Sc. Rev. 208, 81-106 (2017). doi:10.1007/s11214-017-0338-8

16. N. Hirata et al., The spatial distribution of impact craters on Ryugu. Icarus 338, 113527 (2019). doi:10.1016/j.icarus.2019.113527

17. M. Arakawa et al., An artificial impact on the asteroid 162173 Ryugu formed a crater in the gravity-dominated regime. Science 10.1126/science.aaz1701 (2020).

18. W. F. Bottke et al., The fossilized size distribution of the main asteroid belt. Icarus $\mathbf{1 7 5}$, 111-140 (2005). doi: 10.1016/j.icarus.2004.10.026

19. D. P. O'Brien, R. Greenberg, The collisional and dynamical evolution of the main-belt and NEA size distributions. Icarus 178, 179-212 (2005). doi: 10.1016/j.icarus.2005.04.001

20. W. F. Bottke et al., In search of the source of asteroid (101955) Bennu: Applications of the stochastic YORP model. Icarus 247, 191-217 (2015). doi:10.1016/j.icarus.2014.09.046

21. B. Gladman, P. Micheal, C. Froeschlé, The near-Earth object population. Icarus 146, 176189 (2000). doi:10.1006/icar.2000.6391

22. P. Michel, M. Delbo, Orbital and thermal evolutions of four potential targets for a sample return space mission to a primitive near-Earth asteroid. Icarus, 209, 520-534 (2010). doi: 10.1016/j.icarus.2010.05.013

23. M. Delbo, P. Michel, Temperature history and dynamical evolution of (101955) 1999RQ36: a potential target for sample return from a primitive asteroid. Astrophys. J. Lett. 728, L42 (2011). doi:10.1088/2041-8205/728/2/L42

24. D. D. Mazanek et al., Asteroid redirect mission (ARM) formulation assessment and support team (FAST) final report. NASA Technical Memorandum TM-2016-210911, 130 pp.

25. S. Marchi et al., Heating of near-Earth objects and meteoroids due to close approaches to the Sun. Mon. Not. R. Astron. Soc. 400, 147-153 (2009). doi:10.1111/j.1365-2966.2009.15459.x

26. H. Suzuki et al., Initial inflight calibration for Hayabusa2 optical navigation camera (ONC) for science observations of asteroid Ryugu. Icarus 300, 341-359 (2018). doi: 10.1016/j.icarus.2017.09.011

27. S. Kameda et al., Preflight calibration test results for optical navigation camera telescope (ONC-T) onboard the Hayabusa2 spacecraft. Space Sci. Rev. 208, 17-31 (2017). doi: 10.1007/s 11214-015-0227-y

28. E. Tatsumi et al., Updated inflight calibration of Hayabusa2's Optical Navigation Camera (ONC) for scientific observations during the cruise phase. Icarus 325, 153-195 (2019). doi: 10.1016/j.icarus.2019.01.015

29. E. Tatsumi et al., Updated flat-fields of ONC-T/Hayabusa2 based on close encounter with Ryugu. Proc. Lunar Planet. Sci. Conf. 50, abstr. 1745 (2019).

30. J. R. Elliott, Y.-H. Huang, D. A. Minton, A. M. Freed, The length of lunar crater rays explained using secondary crater scaling. Icarus 312, 231-246 (2018). doi:10.1016/j.icarus.2018.04.015

31. S. K. Croft, Cratering flow fields: Implications for the excavation and transient expansion stages of crater formation. Proc. Lunar Planet. Sci. Conf. 11th, 2347-2378 (1980). 
32. E. Tatsumi, S. Sugita, Cratering efficiency on coarse-grain targets: Implications for the dynamical evolution of asteroid 25143 Itokawa. Icarus 300, 227-248 (2018). doi: 10.1016/j.icarus.2017.09.004

33. W. F. Bottke, R. Greenberg, Asteroidal collision probabilities. Geophy. Res. Lett. 20, 879881 (1993). doi:10.1029/92GL02713

34. W. F. Bottke, M. C. Nolan, R. Greenberg, R. A. Kolvoord, Velocity distributions among colliding asteroids. Icarus 107, 255-268 (1994). doi: 10.1006/icar.1994.1021

35. K. A. Holsapple, K. R. Housen, A crater and its ejecta: An interpretation of Deep Impact. Icarus 191, 586-597 (2007). doi:10.1016/j.icarus.2006.08.035

36. G. J. Flynn, Physical properties of meteorites and interplanetary dust particles: clues to the properties of the meteors and their parent bodies. Earth Moon Planets 95, 361-374 (2004). doi:10.1007/s11038-005-9025-y

37. T. Nakamura et al., Irradiation-energy dependence of the spectral changes of hydrous C-type asteroids based on $4 \mathrm{keV}$ and $20 \mathrm{kev}$ He exposure experiments of Murchison CM chondrite. Proc. Lunar Planet. Sci. Conf. 51, abstr. 1310 (2020).

38. M. Matsuoka et al., Pulse-laser irradiation experiments of Murchison CM2 chondrite for reproducing space weathering on C-type asteroids. Icarus 254, 135-143 (2015). doi: 10.1016/j.icarus.2015.02.029

Acknowledgments: The Hayabusa2 spacecraft was developed and built under the leadership of Japan Aerospace Exploration Agency (JAXA), with contributions from the German Aerospace Center (DLR) and the Centre National d'Études Spatiales (CNES), and in collaboration with NASA, Nagoya Univ., Univ. of Tokyo, National Astronomical Observatory of Japan (NAOJ), Univ. of Aizu, Kobe Univ., and other universities, institutes, and companies in Japan. We thank the many engineers, including Noriyasu Inaba at JAXA and Tetsuya Masuda, Seiji Yasuda, Kouta Matsushima, and Takeshi Ohshima at NEC Corp. for their dedicated work on the Hayabusa 2 mission, Koshi Sato at NEC Corp. for ONC development, and Shingo Kashima at NAOJ for optical calculations. We are grateful to B.E. Clark and two anonymous reviewers for thoughtful and constructive comments in their review of this paper. We also thank W. F. Bottke for useful comments on the orbital evolution of NEAs. Funding: T.Mo., S.Su., N.S., S.Ka., M.M., S.Sa., M.Ab., R.N., A.M., K.W., and S.W. was supported by KAKENHI from the Japan Society for the Promotion of Science (JSPS) (Grant Nos. 17H06459, 19H01951, 17H01175, 17KK0097, 19H00727, 18H01267, 18K11610, 19K03955, 16H04044, 19K03958). This study was supported by the JSPS Core-to-Core program "International Network of Planetary Sciences." T.H. acknowledges funding support from NASA EW and PDART programs. M.Hi. acknowledges support from NASA/Solar System $\quad$ Workings (NNH17ZDA001N/80NSSC19K0548) and Auburn University's intramural research grant. O.S.B. acknowledges support from OSIRIS-REx under Contract NNM10AA11C issued through the NASA New Frontiers Program..D.D. and C.M.E. acknowledge funding through the NASA Hayabusa2 Participating Scientist Program. P.M. acknowledge funding from the French space agency CNES and from Academies of Excellence: Complex systems and Space, environment, risk, and resilience, part of the IDEX JEDI of the Université Côte d'Azur, and from the European Union's Horizon 2020 research and innovation programme under grant agreement No 870377 
(project NEO-MAPP). Author contributions: T.Mo. coordinated coauthor contributions, led the ONC data analyses and interpretations, and wrote the paper with contributions from S.Su., N.H. (Aizu), and S.Ka.; ONC data acquisitions and reductions: R.Ho., N.S., Y.Yo., M.Ya., S.Su., T.Mo., S.Ka. H.Sa., M.M., T.K., E.T., C.Ho., K.O., H.Su., K.Yoshio, M.Ha., Y.C., Y.Ii., N.Tak., C.S., K.Yu., M.I., H.Ka., K.Mo., and D.D.; Spectral analysis: E.T., D.D., R.Ho., Y.Yo., S.Su., and T.Mo.; Crater statistics and geomorphology analyses: T.Mo., Y.C., M.K., N.H. (Kobe), O.S.B., C.M.E., E.T., and S.Su.; Shape modeling: H.N. (Kobe), N.H. (Aizu), O.S.B., C.M.E., R.N., Y.S., S.W.; NIRS3 data acquisitions and reductions: K.K., T.N., L.R., C.P., T.Iw., M.Ab., M.Oh., Y.N., and K.T.; Landing site characterization: Y.Ts., S.W., T.Sa., S.Ki., T.Y., N.O., Y.Ya., Y.S., K.S., N.H. (Kobe), K.O., K.K., N.H. (Aizu), K.W., H.Yab., Y.Is, R.N., T.Mo., N.S., K.Ma., H.Se., R.Ho., E.T., Y.Yo., C.Ho., T.Mic., M.M., A.M., N.Tan., Y.F., T.Ir., M.S.; Science operations of spacecraft: T.Miz., C.Hi., S.H., O.M., T.Sh., S.So., R.T., H.Yan., M.Ha., M.Iw., M.Ab., H.Sa., M.Oz., H.T., Y.Ya., T.O., Y.S., K.S., Y.Ii., H.N., S.Ki., T.Y., N.O., G.O., Y.M., K.Yoshik., T.T., Y.Ta., A.F., S.N., F.T., S.Tan., M.Yo., T.Sa., S.W., and Y.Ts.; Project administration: S.W., M.Yo., S.Tac., K.K., S.Su., T.O., N.N., M.Ar., M.Ab., H.I., S.Tan., S.N., F.T., T.Sa., and Y.Ts.; Interpretation and writing contribution: T.Mo., S.Su., R.Ho., E.T., Y.C., Y.Yo., S.Ka., N.S., M.M., M.Ya., N.H. (Kobe), N.H. (Aizu), S.W., T.Mic., M.Hi., T.H., R.He., G.K., S.Sa., T.N., K.W., N.Tan., C.M.E., D.D., and P.M. All authors discussed the results and commented on the manuscript. Competing interests: Y.Ya. is also affiliated with Tokyo Metropolitan University. Data and materials availability: The images, input data and the DEM in the paper are available at the JAXA Data Archives and Transmission System (DARTS) website (http://www.darts.isas.jaxa.jp/pub/hayabusa2/paper/Morota 2020/). Additional data from the mission are delivered to the DARTS archive at https://www.darts.isas.jaxa.jp/planet/project/hayabusa2/, and higher-level data products will be available in the Small Bodies Node of the Planetary Data System https://pdssmallbodies.astro.umd.edu/ one year after departure from the asteroid."

\section{Supplementary Materials:}

Materials and Methods

Supplementary Text

Figs. S1 to S14

Tables S1

Movie S1

References (26-38) 
Figure 1. Spectral slope of Ryugu's surface. (A) Global map of $b-x$ slope $\left(\mu \mathrm{m}^{-1}\right)$, indicated by the color bar, superimposed on a $v$-band image map. The white arrow indicates the location of the first touchdown (TD) point $\left(4.30^{\circ} \mathrm{N}, 206.47^{\circ} \mathrm{E}\right)$. Craters larger than $20 \mathrm{~m}$ in diameter are shown by black circles. Dashed lines indicate areas shown in other panels. (B) $v$-band image (hyb2_onc_20180801_183933_tvf) obtained from $5.1 \mathrm{~km}$ altitude. Yellow arrows indicate craters with bluer interior than the surrounding materials. (C) $b-x$ slope of the same region as panel B. Craters larger than $20 \mathrm{~m}$ in diameter are shown by white dashed circles. Blue craters B1 and B2 are higher in the stratigraphy than red craters R1, R2 and R3. There is no blue crater stratigraphically superposed by red craters, suggesting that the blue craters are younger than the red craters. (D) $v$-band image and (E) $b$ - $x$ slope image showing a 9-meter-sized crater with redder interior than the surrounding materials, close to the touchdown point marked with a white cross. Panels $\mathrm{C}$ and $\mathrm{E}$ are on the same color scale as A.

Figure 2. Touchdown site before, during, and after the touchdown operation. (A) Boulder and crater map around the touchdown site L08-E1. The light blue arrows indicate the location of the target marker $(\mathrm{TM})\left(4.04^{\circ} \mathrm{N}, 206.01^{\circ} \mathrm{E}\right)$ and the touchdown point of the sampler horn (TD) $\left(4.30^{\circ} \mathrm{N}, 206.47^{\circ} \mathrm{E}\right)(7)$. The light blue circle indicates the L08-E1 area. The white dashed circles indicate craters. Boulder heights $(H)$ were estimated from their shadow lengths; those with $H>1.8 \mathrm{~m}$ are outlined in red, and $H>0.65$ in pink. The boulder nicknamed "Turtle Rock" is indicated by the yellow arrow. The white box indicates the region shown in later panels. (B) and (C) $p$ - $b$ ratio images (6) calculated from $b$ - and $p$-band $(0.95 \mu \mathrm{m})$ images obtained during the touchdown rehearsal operation, from two different altitudes (hyb2_onc_20181015_134707_tbf and hyb2_onc_20181015_134655_tpf). The dashed boxes in B indicate regions shown in the other panels. (D) ONC-W1 image during the spacecraft descent before the touchdown (hyb2_onc_20190221_222859_w1f). The dark ragged Turtle Rock and an example of bright boulders with smooth surfaces (BB) are outlined in yellow and cyan dashed lines, respectively. The white dashed box indicates the area shown in panel E. (E) Close-up of the same image, with yellow arrows indicating fresh bright spots at corners and a possible broken plane of boulders. (F) and (G) ONC-W1 images obtained about 7 and 47 seconds after the touchdown, showing debris lifted from the surface (hyb2 onc 20190221 $222917 \mathrm{w} 1 \mathrm{f}$ and hyb2_onc_20190221_222957_w1f). (H) ONC-T ul-band $(0 . \overline{3} 9 \mu \mathrm{m})$ image obtained at $76 \mathrm{~m}$ altitude after the touchdown (hyb2_onc_20190221_223156_tuf). Turtle Rock was lifted clear of the surface by the exhaust from Hayabusa2's RCS thrusters, indicated by the yellow arrows in panels F-H.

Figure 3. Crater statistics on Ryugu. Histograms of the differences in $b$ - $x$ slopes between the interior and the surroundings of craters larger than $10 \mathrm{~m}$ in diameter, in the latitude ranges (A) $\pm 50^{\circ}$, (B) $\pm 10^{\circ}$, and (C) $-50^{\circ}$ to $-10^{\circ}$ and $10^{\circ}$ to $50^{\circ}$. Bar colors are illustrative only. (D) Crater size-frequency distribution (CSFD) in the latitude range of $\pm 50^{\circ}$. Black, red and blue squares indicate crater frequencies of all craters, red craters (defined as having a difference of $b-x$ slope of $<0.025$ ) and blue craters (difference of $b-x$ slope of $>0.025$ ), respectively. Gray curves indicate cratering chronology models fitted to the data for red and blue craters. The dashed line indicates the empirical saturation level. Error bars are calculated by $\pm N^{1 / 2} / A$, where $N$ is the cumulative number of craters and $A$ is the area of the latitude range of $\pm 50^{\circ}$. The resulting model 
ages for the main belt asteroid (MBA) and near-Earth asteroid (NEA) impact rates are indicated in red and blue text.

Figure 4. A schematic illustration of our suggested evolution of Ryugu. Surface reddening occurred within a short period after the emplacement of red craters and before the formation of blue craters. We interpret the cause of the surface reddening as solar heating while Ryugu came temporally closer to the Sun than at present. Between the formation of Ryugu's spinning topshape and the surface reddening we estimate a time of $9 \mathrm{Ma}$, based on the CSFDs of red craters. From the CSFDs of blue craters, the age of the surface reddening is estimated to be $0.3 \mathrm{Ma}$ using the main-belt collision frequency model and 8 Ma using the NEA collision frequency model. We interpret these as upper and lower limits of age estimates of the surface reddening. After the surface reddening, the redder materials were disrupted and redistributed by impacts, thermal fatigue and mass wasting from the equator to mid latitude regions. A layer of mixed red and blue material subsequently formed on Ryugu's surface. 


\section{Science \\ MIAAAS}
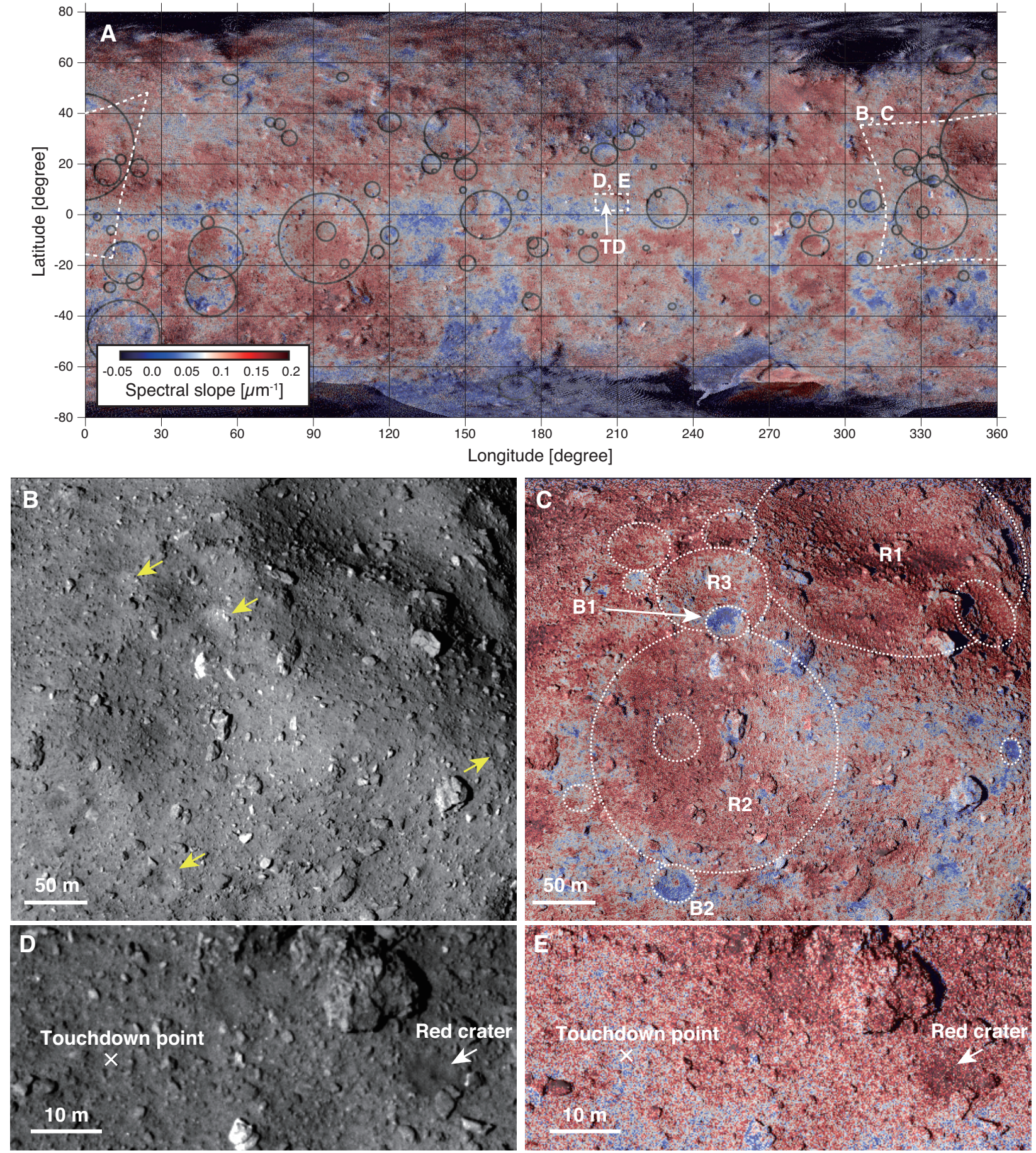


\section{Science \\ MIAAAS}

Submitted Manuscript: Confidential
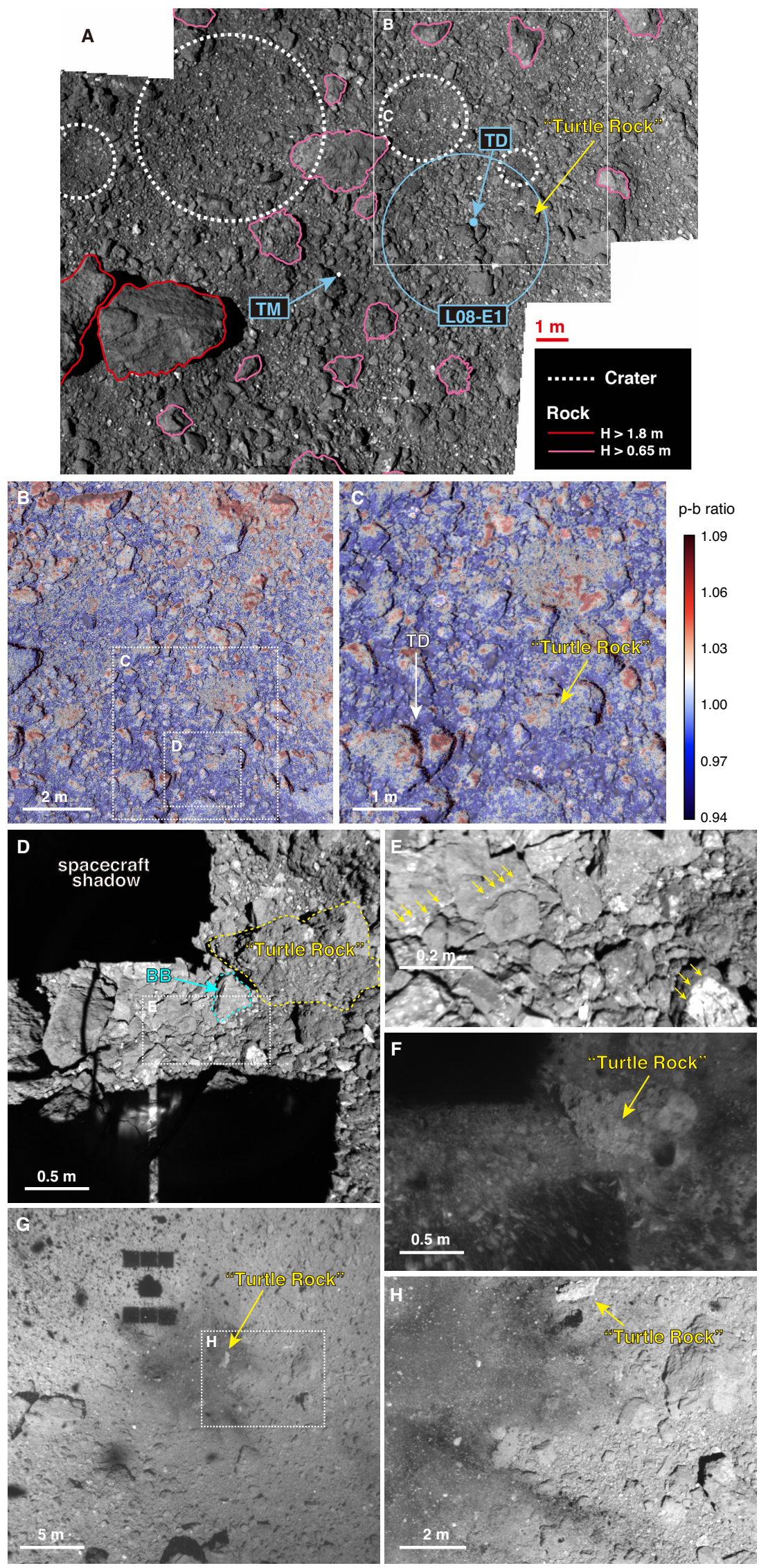
DIAAAS
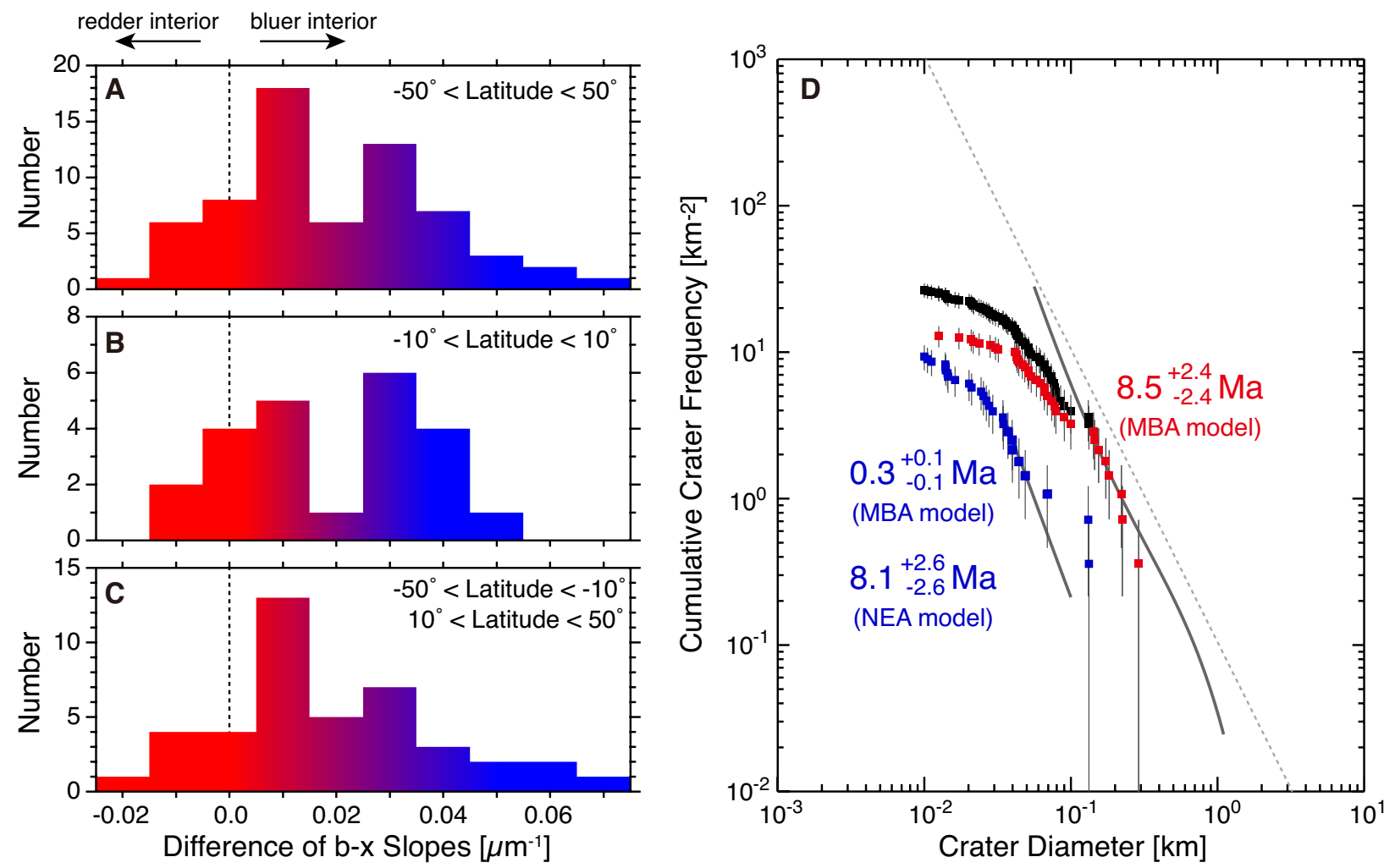


\section{Science \\ MTAAAS}

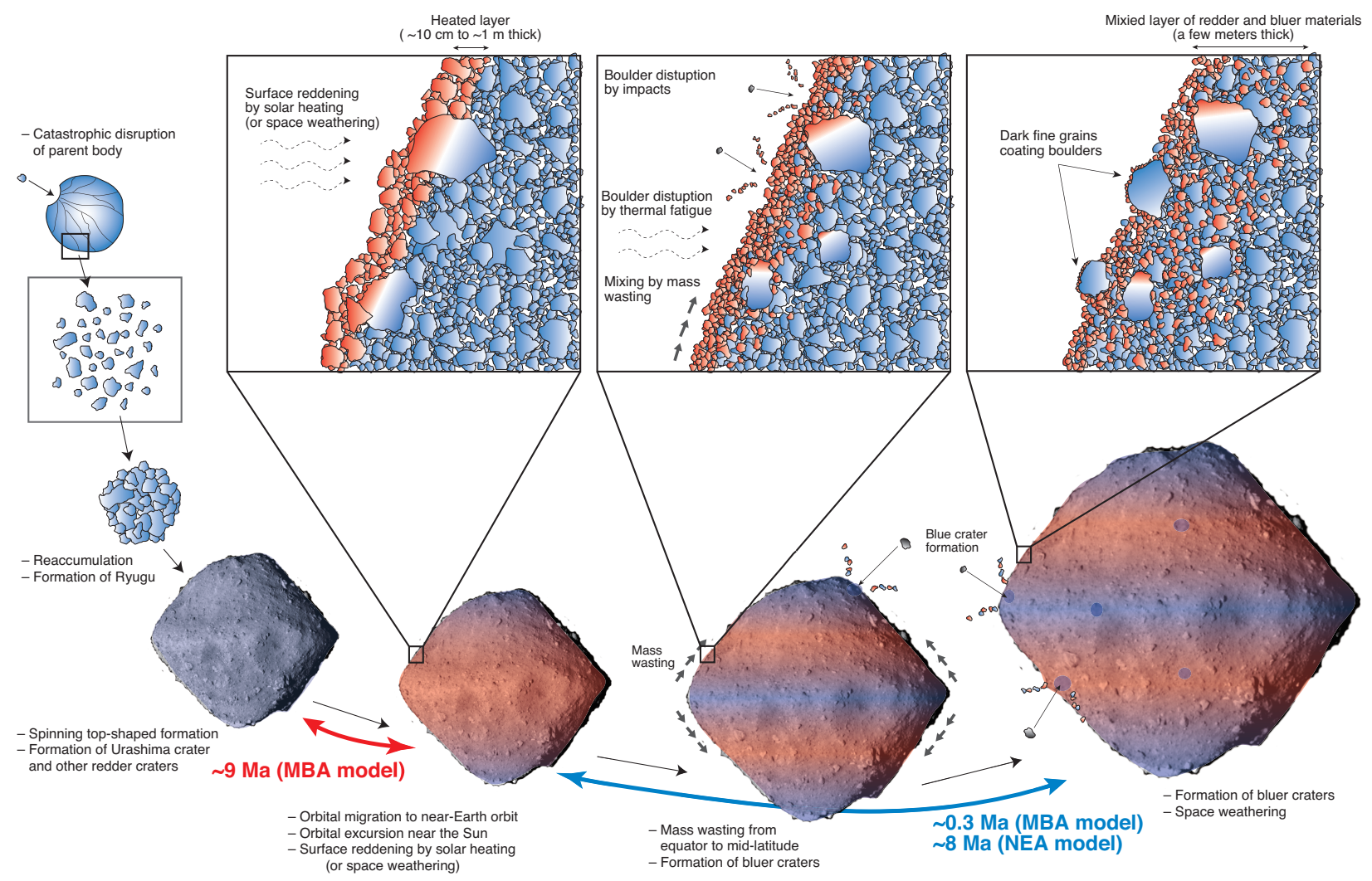

